SCIENTIFIC REPORT

\title{
A protocol for low contamination risk of autologous serum drops in the management of ocular surface disorders
}

\author{
R Lagnado, A J King, F Donald, H S Dua
}

Br J Ophthalmol 2004;88:464-465. doi: 10.1136/bjo.2003.025528

\begin{abstract}
Aim: To assess microbial contamination of $20 \%$ autologous serum (AS) eye drops used in a hospital inpatient setting. Method: 14 patients received autologous serum drops from 4 to 14 days with a cumulative total of 67 days. For each day the first and last drop (total 134 samples) was cultured on broth and blood agar.

Results: Four patients (9 samples) grew Staphylococcus epidermidis only. One patient (1 sample) showed Staphylococcus epidermidis and a scanty growth of viridans streptococci in the same sample, and on different days the same patient grew Staphylococcus aureus in one sample and Staphylococcus epidermidis in another sample. One patient (1 sample) grew micrococcus. There was no clinical or microbial evidence of infection in any of these six patients Conclusion: This study shows that autologous serum drops can be safely used in an inpatient setting, under a strict protocol of preparation and storage, without significant risk of bacterial contamination and consequent infection.
\end{abstract}

A utologous serum (AS) eyedrops have been reported to be efficacious in the management of ocular surface disorders and following ocular surface reconstruction. ${ }^{1-8}$ As autologous serum drops have a high protein content there is a significant risk of microbial colonisation. The toxicity of any added preservatives can negate some of the beneficial effects of AS and are therefore avoided.

The drops are prepared under sterile conditions on an individual patient basis but there are risks for contamination during the preparation, storage, and use of the drops. When used following ocular surface surgery, they are usually combined with topical prophylactic antibiotics. However, there have been reports of serious infection following use of serum drops. ${ }^{35}$

With the increasing use of autologous serum drops it is important to have information on their sterility. It is also possible that because serum itself may have some bacteriostatic effects-for example, lysosyme, complement, and IgG - the addition of a further bacteriostatic agent may not be necessary.

We conducted a study to establish if contamination of $20 \%$ autologous serum drops, prepared under sterile conditions, occurs over a 24 hour period of one to two hourly use in a hospital inpatient setting.

\section{MATERIALS AND METHODS \\ Patients}

The autologous serum drops used in the management of 14 consecutive patients following ocular surface surgery were examined. No patient had bilateral treatment. Three patients had undergone stem cell transplants, seven amniotic membrane transplants, one tenoplasty and amniotic membrane transplant, and one patient combined penetrating keratoplasty amniotic membrane transplant and stem cell transplant. The patients' age range was $28-84$ years. The frequency of application of drops was one to two hourly for a range of 4 days to two weeks with a cumulative total of 67 days. The end point for the use of serum drops was a stable intact epithelium. The protocol adhered to the tenets of the Declaration of Helsinki, and informed consent was obtained from all participants. Once a bottle was thawed for use it was kept stored in a fridge at $4^{\circ} \mathrm{C}$ between applications.

\section{Preparation of autologous serum drops}

Autologous serum for use as eyedrops was prepared by obtaining approximately $30 \mathrm{ml}$ of the patient's blood in sterile vacutainer tubes without additive. The blood was left to clot at $4{ }^{\circ} \mathrm{C}$ for 10 to 12 hours before centrifuging at 4500 rpm for fifteen minutes. The serum was separated in a laminar flow cabinet and diluted with sterile saline $(0.9 \%)$ to a $20 \%$ concentration and aliquoted in $2.0 \mathrm{ml}$ portions clearly labelled with the patient's details. The vials were kept frozen at $-20^{\circ} \mathrm{C}$. A fresh bottle was thawed and used for treatment every 24 hours. All patients were on topical preservative free chloramphenicol drops.

On the day of use the autologous serum was thawed, aspirated through the rubber cap of the sealed vial with a hypodermic needle and syringe, and transferred to a sterile dropper bottle. One sample for culture was taken following transfer of the solution before any drop was administered to the patient. The second sample was taken before discarding the bottle of serum at the end of 24 hours of use. On each occasion a drop of serum was placed on the centre of a blood agar plate and one drop into a tube of nutrient broth. Specimens were incubated at $37^{\circ} \mathrm{C}$ aerobically for 48 hours. Any growth of organisms in either of the two media was further analysed for identification of the organism(s) grown. This was repeated for each patient on a minimum of four consecutive days and maximum of 14 days of treatment.

\section{RESULTS}

No patients had any adverse effects such as superficial punctate keratitis, epithelial sloughing, delayed epithelial healing, or stromal melting from the use of $20 \%$ autologous serum. In all patients a successful outcome, defined by rapid and complete epithelial healing, was observed. Of the 134 samples cultured only 13 were positive as outlined in the flow diagram (fig 1). Staphylococcus epidermidis, a skin commensal, grew in 11 samples of five patients. In four of these 11 samples the organism(s) grew in broth only. One of these four was a sample before use on the patient. Interestingly the drop taken at the end of 24 hours use from the same bottle

Abbreviation: AS, autologous serum 


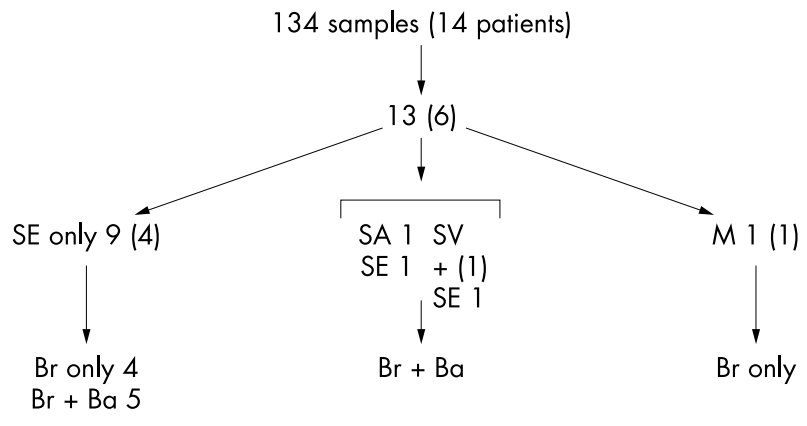

Figure 1 Culture results for the samples tested. Numbers in parenthesis indicate patient number. SE, Staphylococcus epidermidis; SV, viridans streptococcus; SA, Staphylococcus aureus; $M$, micrococcus; $B r$, enriched broth; $\mathrm{Ba}$, blood agar.

did not show any microbial growth. The other three of the four positive in broth only were samples from bottles at the end of 24 hours of use. The remaining seven of these 11 samples grew S epidermidis on blood agar as well. Two of these were from the same bottle of serum, which was culture positive at the start and end of 24 hours of use. A further five samples, which had been negative before use, became culture positive after 24 hours of use. In one of these samples there was additional scanty growth of viridans streptococci. For the same patient, on a different day a sample (end of 24 hours of treatment) showed $S$ aureus. In a further, sixth patient, a single starting sample grew micrococcus, a coagulase negative staphylococcal skin commensal.

In none of the six patients who received autologous serum drops from which organisms were grown was there any clinical or microbiological evidence of infection. All patients were also on prophylactic preservative free chloramphenicol drops and this may have contributed to the lack of infection observed.

\section{DISCUSSION}

This is the first study to specifically examine the contamination of autologous serum drops used in a hospital inpatient setting. We used 20\% autologous serum because earlier reports have confirmed that at this concentration it is effective in the treatment of dry eyes ${ }^{3}$ and persistent epithelial defects. ${ }^{4}$ This concentration gives adequate viscosity and a relatively high concentration of growth factors that are stable at $4^{\circ} \mathrm{C}$ for 1 month. ${ }^{4}$

There does not appear to be a need to add a bacteriostatic agent or preservative to serum when using the protocol for preparation and treatment regime described in this study. We have shown that there is no significant microbial contamination after 24 hours of use in a hospital inpatient setting. Only one out of a total of 134 samples grew a significant ocular surface pathogen, Staphylococcus aureus. This was grown after a bottle of autologous serum had been in use for 24 hours. None of the bottles grew any fungi (in the enriched broth) after 24 hours of use. Eleven samples tested in five of the patients did grow $S$ epidemidis and in one of these there was scanty growth of viridans streptococcus as well. The growth of $S$ epidermidis in four samples in enriched broth only, with no growth on the blood agar plate indicates a very low level of contamination. This was found in both first and second samples indicating no significant increase in colonisation with use through a 24 hour period.

Another sample in a sixth patient grew micrococcus on a morning sample only. Like $S$ epidermidis, micrococcus is a common skin commensal and neither are considered virulent ocular surface pathogens. We cannot exclude the possibility that these were contaminants at the time of inoculation of the blood agar plate or broth. This could explain why in one patient the same bottle of serum was positive for $S$ epidermidis before use but negative at the end of 24 hours use. Alternatively, this may reflect bacteriostatic or cidal properties in serum. S epidermidis is a potentially serious intraocular pathogen, and use of autologous serum after ocular surface reconstructive procedures that have been combined with intraocular surgery should be carefully considered.

Repeated use of drops from the same bottle with storage in the fridge may increase the risk of bacterial and fungal contamination. A previous study has reported on autologous serum growing the same organism as that which was responsible for a keratitis in two out of 22 patients. ${ }^{5}$ The bottles of serum were used for a week at a time and then discarded and were not routinely cultured, but were when microbial keratitis was identified on clinical grounds. In one case candida was the causative pathogen also found in the serum and in another case, a coagulase negative Staphylococcus. In a different study on the use of autologous serum in 12 dry eye patients, ${ }^{3}$ serum bottles were also used for a week at a time and then returned for culture, and it was found that mixed organisms and yeast had grown in one bottle but there was no conjunctivitis or keratitis in the patient. It shows that the adopted protocols, both for preparation and administration of autologous drops, allow safe use of the drops in a hospital setting. This study demonstrates that under the protocol described, it is safe to use autologous serum drops for up to 24 hours, in a hospital inpatient setting.

\section{Authors' affiliations}

R Lagnado, A J King, H S Dua, Division of Ophthalmology and Visual Sciences, University of Nottingham, UK

F Donald, Department Microbiology, University of Nottingham, UK

Commercial relationships of authors: none. Ruby Lagnado was the Vision Express Fellow, Corneal and Contact Lenses, University of Nottingham.

Correspondence to: Professor H S Dua, Division of Ophthalmology and Visual Sciences, B Floor, Eye Ear Nose Throat Centre, University Hospital, Queens Medical Centre, Nottingham NG7 2UH, UK; harminder.dua@nottingham.ac.uk

Accepted for publication 1 September 2003

\section{REFERENCES}

1 Fox R, Chan R, Michelson J, et al. Beneficial effect of artificial tears made with autologous serum in patients with keratoconjunctivitis sicca. Arthritis Rheum 1984;27:459-61.

2 Tsubota K, Goto E Fujita $\mathrm{H}$, et al. Treatment of dry eye by autologous serum application in Sjogren's syndrome. Br J Ophthalmol 1999;83:390-5.

3 Tananuvant N, Daniell M, Sullivan L J, et al. Controlled study of the use of autologous serum in dry eye patients. Cornea 2001;20:802-6.

4 Tsubota K, Goto E, Shimmurma S, et al. Treatment of persistent epithelial defect by autologous serum application. Ophthalmology 1999;106:1984-9.

5 Poon AC, Geerling G, Dart JKG, et al. Autologous serum eyedrops for dry eyes and epithelial defects: clinical and in vitro toxicity studies. Br J Ophthalmol 2001;85: 1188-97.

6 Tsubota K, Satake Y, Ohyama M, et al. Surgical reconstruction of the ocular surface in advanced ocular cicatricial pemphigoid and Stevens-Johnson syndrome. Am J Ophthalmol 1996;122:38-52.

7 Dua HS, Azuara-Blanco A. Allo-limbal transplantation in patients with limbal stem cell deficiency. Br J Ophthalmol 1999:83:414-19.

8 Goto E, Shimmura S, Shimazaki J, et al. Treatment of superior limbic keratoconjunctivitis by application of autologous serum. Cornea 2001;20:807-10.

9 Ogawa Y, Okamoto S, Mori T, et al. Autologous serum eye drops for the treatment of severe dry eyes in patients with chronic graft-versus-host disease. Bone Marrow Transplant 2003;31:579-83. 